\title{
Comment faire évoluer le paradigme du développement clinique et son découpage en phases I, II, III
}

\author{
Marion Bamberger ${ }^{1}$, Nicholas Moore ${ }^{2}$, Philippe Lechat ${ }^{3}$ et les participants à la Table Ronde $N^{\circ} 3$ de Giens XXVI \\ 1 Laboratoire Bristol Myers Squibb, Rueil Malmaison, France \\ 2 Service de Pharmacologie, Université Victor Segalen, Bordeaux, France \\ 3 Afssaps, Saint Denis, France
}

Texte reçu le 27 avril 2011 ; accepté le 10 mai 2011

\footnotetext{
Mots clés : développement clinique des médicaments ; recherche clinique ; phase I ; phase II ; phase III ; phase IV ; réglementaire ; design adaptatif
}

Résumé - Partant de la constatation que depuis 30 ans, le coût du développement augmente régulièrement à mesure que le nombre de nouvelles entités chimiques atteignant le marché décroît, comment faire des «économies » en termes de développement clinique, l'objectif étant un accès plus rapide au médicament, pour les besoins médicaux non couverts ? Plusieurs instruments existent pour permettre une mise à disposition plus rapide de produits innovants : les autorisations temporaires d'utilisation ou ATU (qui ne sont pas concernées par ce travail), les autorisations de mise sur le marché (AMM) conditionnelles, les AMM sous circonstances exceptionnelles.

Ces éléments sont repris dans la «road map » de l'Agence européenne du médicament, (European Medicines Agency ou EMA), qui dit : «A key issue for Regulators will be if a more "staggered" approval should be envisaged, characterised by a better defined/more restricted population of good responders, followed by a broadening of the population postauthorisation when more "real life" data are available. In addition, maximising the value of information generated in the post-authorisation phase should be developed through the use of cohorts and other prospectively collected use data, especially in the case of conditional marketing authorisations. »

Le règlement intérieur de la Commission de transparence pour sa part prévoit la notion d'examen préliminaire : afin de préparer au mieux l'instruction des dossiers des produits présumés innovants et de limiter les délais, le bureau peut engager une étude préliminaire dès le dépôt du dossier au Comité des spécialités pharmaceutiques humaines (CHMP). Il peut à cette occasion solliciter de la firme des éléments d'information et faire appel à des experts externes. La mise en œuvre de cette étude préliminaire n'exonère pas la firme de l'obligation de déposer un dossier complet.

Les études post inscription demandées par la commission de transparence (ISPEP) le sont souvent dans le cas d'hésitations sur le niveau d'amélioration du service médical rendu ou ASMR (niveau II/III ou IV/V). Ces demandes concernent essentiellement des incertitudes sur la transposabilité, le profil patient ou le respect du bon usage. Parmi les études dont les résultats ont été rendus, dans 15 cas les résultats sont en conformité avec les attentes, dans 6 cas elles ont abouti à des réévaluations à la baisse, enfin dans 3 cas elles étaient non conclusives. Les recommandations finales de la Table Ronde étaient :

- définition du besoin médical non couvert à travailler en concertation (industriels et autorités de Santé) ;

- prévision d'un Plan d'investigations complémentaires (PIC) au delà de l'AMM très tôt pour conforter AMM précoce, et/ou préparation HTA et le suivre (actions contraignantes possibles);

- utilisation accrue des techniques de modélisation et de leur transposabilité ;

- «intussusception » des phases pour optimiser le développement d'un dossier complet ;

- «avis scientifiques » précoces (EMA, Agence française de sécurité sanitaire des produits de santé (Afssaps), Haute autorité de santé (HAS));

- sensibilisation des autorités, industriels, médecins et patients aux études observationnelles contrôlées ;

- développement de l'exploitation des bases de données publiques.

\footnotetext{
* Pour la liste des participants, voir en fin d'article
} 


\section{Objectif de la Table Ronde}

Partant de la constatation que depuis 30 ans, le coût du développement augmente régulièrement à mesure que le nombre de nouvelles entités chimiques atteignant le marché décroît, comment faire des «économies » en termes de développement clinique, l'objectif étant un accès plus rapide au médicament, pour les besoins médicaux non couverts?

\section{Situation actuelle}

Plusieurs instruments existent pour permettre une mise à disposition plus rapide de produits innovants :

- Les autorisations temporaires d'utilisation ou ATU (qui ne sont pas concernées par ce travail).

- Les autorisations de mise sur le marché ou AMM sous circonstances exceptionnelles, qui correspondent aux cas où il manque des études dans les conditions normales d'utilisation (données d'efficacité et de sécurité d'emploi), quand le titulaire d'AMM justifie d'une indication rare ou d'une petite population cible, de l'absence d'informations disponibles en l'état des connaissances scientifiques, et où des considérations éthiques rendent difficile la collection des données manquantes. Un avis scientifique sera demandé 4 à 6 mois avant la soumission. Des obligations spécifiques peuvent être demandées par les autorités d'enregistrement : plan de gestion des risques, études post-mise sur le marché (post-MM), ainsi que l'indication dans le résumé des caractéristiques du produit (SPC) et dans l'information à l'utilisateur (PIL) de la nature de l'AMM. Il peut également être demandé des conditions particulières d'utilisation ou de dispensation.

- les AMM conditionnelles, qui sont accordées sous quatre conditions : un rapport bénéfice-risque favorable (sur des critères de substitution, dans des sous-populations, ou sur des analyses intermédiaires), des données cliniques à compléter (en particulier dans des sous-populations), l'existence d'un besoin médical non couvert, et que le bénéfice de santé publique soit supérieur aux risques, justifiant d'une mise à disposition avant la complétion du dossier habituel. Il faut cependant que toutes les données réglementaires relatives à la qualité et les données non cliniques soient disponibles (sauf situations d'urgence), que la nature conditionnelle de l'AMM soit mentionnée dans le SPC et la notice d'information au patient. Cette AMM est octroyée pour un an, renouvelable.

Ces éléments sont repris dans la «road map» de l'Agence européenne du médicament, (European Medicines Agency ou EMA), qui dit : "A key issue for Regulators will be if a more "staggered" approval should be envisaged, characterised by a better defined/more restricted population of good responders, followed by a broadening of the population post-authorisation when more "real life" data are available. In addition, maximising the value of information generated in the post-authorisation phase should be developed through the use of cohorts and other prospectively collected use data, especially in the case of conditional marketing authorisations. »

Le règlement intérieur de la Commission de Transparence pour sa part prévoit la notion d'examen préliminaire : afin de préparer au mieux l'instruction des dossiers des produits présumés innovants et de limiter les délais, le bureau peut engager une étude préliminaire dès le dépôt du dossier au comité des spécialités pharmaceutiques humaines (CHMP). Il peut à cette occasion solliciter de la firme des éléments d'information et faire appel à des experts externes. La mise en œuvre de cette étude préliminaire n'exonère pas la firme de l'obligation de déposer un dossier complet.

Les études post inscription demandées par la commission de transparence le sont souvent dans le cas d'hésitations sur le niveau d'amélioration du service médical rendu (ASMR) [niveau II/III ou IV/V]. Ces demandes concernent essentiellement des incertitudes sur la transposabilité, le profil des patient ou le respect du bon usage en utilisation réelle. Parmi les études dont les résultats ont été rendus, dans 15 cas les résultats étaient en conformité avec les attentes, dans 6 cas les résultats ont abouti à des réévaluations à la baisse, enfin dans 3 cas ils étaient non conclusifs.

\section{Propositions de la Table Ronde}

Dans ces conditions, la Table Ronde émet des propositions ou des pistes de réflexion sur des leviers d'action pour répondre aux objectifs d'accélération et de simplification du développement.

Certaines de ces stratégies sont générales et s'appliquent à tous les dossiers :

- élargir les phases I aux sujets malades (phase I/II);

- développer des phases II comparatives (phase II-III) ; ${ }^{[1]}$

- optimiser le choix des doses, par modélisation, ou en déterminant au mieux la dose biologique efficace;

- utiliser des biomarqueurs validés ; ${ }^{[2]}$

- enfin, évaluer la possibilité d'utiliser les méthodes adaptatives d'essais cliniques, ${ }^{[3-17]}$ en prévoyant dans le protocole la poursuite du suivi en ouvert des patients dont l'essai a été arrêté prématurément pour efficacité, afin de compléter les données de sécurité.

D'autres stratégies doivent être adaptées aux situations :

- par exemple, en cas de besoin médical majeur non couvert, octroi d'une AMM précoce sur données limitées, mais avec un développement optimisé, et un plan d'investigation complémentaire (PIC) à développer après la mise sur le marché ; 
- dans le cas du premier d'une nouvelle classe, dossier d'AMM «classique» avec la mise en œuvre de leviers d'action tels que l'utilisation de méthodes adaptatives, une optimisation de l'utilisation des données pharmacocinétique/pharmacodynamie (PK/PD);

- lorsqu'il s'agit du troisième ou plus d'une classe ayant d'autres membres déjà commercialisés et bien connus, on devrait pouvoir capitaliser sur l'expérience de la classe : fautil refaire le développement entier, en particulier pour ce qui concerne l'efficacité dans les domaines couverts par des biomarqueurs validés? Ceci ne peut en rien exonérer d'une évaluation stricte de la sécurité de chaque produit qui peut être différente de celle de la classe; on peut également suggérer la mise en œuvre et la participation à des études post inscription de classe (études par pathologie), en développant soigneusement les méthodes d'inclusion de nouveaux produits dans les études en cours, en fonction de leur mise sur le marché. Ceci peut être plus simple dans des études épidémiologiques par pathologie que s'il s'agit de cohortes ad hoc basées sur l'exposition aux produits.

- En cas d'extension d'indication d'un produit déjà commercialisé :

- s'il s'agit du même ciblage moléculaire tel que par exemple des facteurs de croissance non spécifiques (VEGF) dans différents types de cancers : il est indispensable de mettre en place une étude de preuve de concept, puis l'on pourra utiliser les données connues;

- si l'on cible une nouvelle indication avec des caractéristiques de population cible similaires, on pourra prendre en compte les données de sécurité préexistantes (exemple développement d'un produit anticancéreux ciblé dans un autre type de cancer);

- si les caractéristiques de la population cible sont différentes, il sera indispensable de développer des données solides sur la sécurité dans cette nouvelle population-cible (par exemple utilisation de produits ciblés dans des populations non cancéreuses).

\section{Les recommandations finales de la Table Ronde}

Les recommandations finales de la Table Ronde étaient :

- Poursuivre la définition du besoin médical non couvert à travailler en concertation entre industriels et autorités de santé, et identifier les besoins médicaux encore non couverts, afin d'anticiper sur des possibilités de développement facilité ou accéléré.
- Prévoir un plan d'investigations complémentaires (PIC) au delà de l'AMM très tôt afin de conforter une AMM précoce.

- Préparer et suivre l'évaluation de la valeur ajoutée du produit (health technology assessment ou transparence), pouvant aboutir à des actions contraignantes avant ou après la mise sur le marché.

- Utilisation accrue des techniques de modélisation et de leur transposabilité en situation réelle d'utilisation post-MM.

- «Intussusception» ou intégration des phases successives de développement clinique pour optimiser le développement d'un dossier complet.

- Avis scientifiques précoces ((EMA, Agence française de sécurité sanitaire des produits de santé (Afssaps), Haute autorité de santé (HAS)).

- Sensibiliser les autorités, industriels, médecins et patients aux études observationnelles contrôlées.

- Développer l'exploitation des bases de données publiques.

\section{Participants}

Michel Azizi (CIC, HEGP Paris), Patrick Blin (Pharmacologie Bordeaux), Myriam Bouhassira (Lilly France), Dominic Cellier (Merck Serono), Jean-Paul Demarez (Pierre Fabre Médicaments), Xavier Duval (Inserm AP-HP Paris), François Gueyffier (CHU Lyon), Claire Le Jeunne (Hotel Dieu, Paris), Christian Libersa (CIC, CHRU Lille), Florence Mahlberg-Gaudin (Servier), Patrick Maison (CHU Henri Mondor, Paris), Pierre Marquet (Hôpital Dupuytren, Limoges), Mathieu Molimard (CHU, Bordeaux), Aurélie Moser (Roche), Mira Pavlovic (HAS), Pascal Piedbois (Astra Zeneca), Odile Regnier (CNAMTS), Jean-Charles Reynier (AP-HM, Marseille), Catherine Rey-Quinio (Afssaps), Patrick Rossignol (CIC, CHU Vandoeuvre les Nancy), Alain Spriet (Alain Spriet Conseil), Franck Vignal (Sanofi Aventis).

\section{Références}

1. Jenkins M, Stone A, Jennison C. An adaptive seamless phase II/III design for oncology trials with subpopulation selection using correlated survival endpointsdagger. Pharm Stat 2010 Dec 8

2. Eickhoff JC, Kim K, Beach J, et al. A Bayesian adaptive design with biomarkers for targeted therapies. Clin Trials 2010 Oct; 7(5): 546-56

3. Benda N, Brannath W, Bretz F, et al. Perspectives on the use of adaptive designs in clinical trials. Part II. Panel discussion. J Biopharm Stat 2010 Nov; 20(6): 1098-112

4. Brannath W, Burger HU, Glimm E, et al. Comments on the draft guidance on "adaptive design clinical trials for drugs and biologics" of the U.S. Food and Drug Administration. J Biopharm Stat 2010 Nov; 20(6): 1125-31

5. Chang M. Introduction to discussion papers on draft FDA guidance on adaptive designs. J Biopharm Stat 2010 Nov; 20(6): 1113-4 
6. Cheng B, Chow SC. On flexibility of adaptive designs and criteria for choosing a good one-a discussion of FDA draft guidance. J Biopharm Stat 2010 Nov; 20(6): 1171-7

7. Chow SC. A note on special articles on adaptive clinical trial designs. J Biopharm Stat 2010 Nov; 20(6): 1088-9

8. Chuang-Stein C, Beltangady M. FDA draft guidance on adaptive design clinical trials: Pfizer's perspective. J Biopharm Stat 2010 Nov; 20(6): 1143-9

9. Cook T, DeMets DL. Review of draft FDA adaptive design guidance. J Biopharm Stat 2010 Nov; 20(6): 1132-42

10. Emerson SS, Fleming TR. Adaptive methods: telling "the rest of the story". J Biopharm Stat 2010 Nov; 20(6): 1150-65

11. Fu H, Manner D. Bayesian adaptive dose-finding studies with delayed responses. J Biopharm Stat 2010 Sep; 20(5): 1055-70

12. Gallo P, Anderson K, Chuang-Stein C, et al. Viewpoints on the FDA draft adaptive designs guidance from the PhRMA working group. J Biopharm Stat 2010 Nov; 20(6): 1115-24
13. Liu Q, Chi GY. Understanding the FDA guidance on adaptive designs: historical, legal, and statistical perspectives. J Biopharm Stat 2010 Nov; 20(6): 1178-219

14. Lu Q, Tse SK, Chow SC. Analysis of time-to-event data under a two-stage survival adaptive design in clinical trials. J Biopharm Stat $2010 \mathrm{Jul}$; 20(4): 705-19

15. Wang SJ. Editorial. Adaptive designs: appealing in development of therapeutics, and where do controversies lie? J Biopharm Stat 2010 Nov; 20(6): 1083-7

16. Wang SJ. Perspectives on the use of adaptive designs in clinical trials. Part I. Statistical considerations and issues. J Biopharm Stat 2010 Nov; 20(6): 1090-7

17. Zhou Y. Adaptive designs for Phase I dose-finding studies. Fundam Clin Pharmacol 2010 Apr; 24(2): 129-38

Correspondance et offprints : Nicholas Moore, Service de Pharmacologie, Université Victor Segalen, Zone Nord, Bat 1A, CHU de Bordeaux, 33076 Bordeaux Cedex, France.

E-mail : nicholas.moore@pharmaco.u-bordeaux2.fr 